\title{
What has changed in kidney transplantation in small islands in Japan? Experience in our center
}

\author{
Hajime Hirano ${ }^{1 *}$ D, Ryoichi Maenosono ${ }^{1}$, Tomohisa Matsunaga $^{1}$, Hirofumi Uehara', Hayahito Nomi', \\ Takuya Tsujino', Naoki Tanda', Kenkichi Saito ${ }^{1}$, Taizo Uchimoto ${ }^{1}$, Naokazu Ibuki', Teruo Inamoto', \\ Yoshihiro Tokeshiªnd Haruhito Azuma'
}

\begin{abstract}
Background: Okinoerabu Island and Tokunoshima Island lie in the sea to the south of the Japanese mainland, about $100 \mathrm{~km}$ north of Okinawa and about $500 \mathrm{~km}$ south of Kyushu. There are no facilities that specialize in kidney transplants, so the patients need to leave the island to undergo the procedure. Up to a few years ago, there were less than five kidney transplant patients on the island. We report the status of transplant medicine on these remote islands, including concrete methods for periodic examinations and how emergencies are handled.

Case presentation: Recipient age was $60.0 \pm 8.9$ years (mean \pm SD); 15 were males and 10 were females. Donor age was $57.9 \pm 8.48$ years (mean \pm SD); 14 were males and 11 were females. Recipient diseases leading to ESRD were diabetes (36. $0 \%)$, chronic glomerulonephritis (28.0\%), and ADPKD (12.0\%). The duration of dialysis prior to transplantation was 382. $6 \pm 233.2$ days (mean \pm SD). We physicians specializing in kidney transplants formed an alliance with local facilities a few years back to create specialized outpatient facilities, and the number of transplant patients has gradually increased. Delayed graft function was observed in only one patient, biopsy-proven acute rejection in four patients, and chronic allograft nephropathy in two patients. In these cases, the local doctor performed the treatment in their facilities under our direction. Most of the treatments were performed safely and successfully.

The mean follow-up period was $1208 \pm 1809$ days. None of the patients has had graft loss, with mean SCr (serum Cr level) of $1.35 \pm 0.85 \mathrm{mg} / \mathrm{dl}$.

Conclusions: To coordinate medical care recipients with their primary care physicians, physicians specializing in kidney transplants no longer need to travel long distances to receive follow-up outpatients. Recently, likelihood of kidney transplantation has been much higher among these islands. The number of transplant patients has gradually increased.
\end{abstract}

Keywords: Remote islands, Kidney transplantation

\section{Background}

In renal replacement surgery in a rural island with limited medical resources, promotion of a medical treatment system for kidney transplant is vital and has been awaited. However, there have been few published documents regarding this issue for our reference. We had been aware of such barriers, so we physicians specializing in kidney transplants formed an alliance with local facilities a few

\footnotetext{
* Correspondence: hajime0407@yahoo.co.jp

'Department of Urology, Osaka Medical College, 2-7 Daigaku-machi,

Takatsuki-shi, Osaka 569-8686, Japan

Full list of author information is available at the end of the article
}

years back to create specialized outpatient facilities, and the number of transplant patients has gradually increased. Recently, the likelihood of kidney transplantation has been much higher among these islands.

We report the status of transplant medicine in those remote islands.

\section{Case presentation}

We studied adult patients living on Okinoerabu and Tokunoshima Islands who received a transplant surgery on the mainland of Japan. The number of transplant patients on both islands was 25 (Table 1). 
Table 1 Clinical characteristics of patients

\begin{tabular}{ll}
\hline & Mean $(n=25)$ \\
\hline Recipient factors & \\
Age at kidney transplantation (years) & $56.3 \pm 11.39$ \\
Gender (M/F) & $15 / 10$ \\
The recipient diseases leading to ESRD & \\
Diabetes mellitus & $9(36.0 \%)$ \\
Chronic glomerulonephritis & $7(28.0 \%)$ \\
ADPKD & $3(12.0 \%)$ \\
RPGN & 2 \\
Nephrosclerosis & 1 \\
Unknown & 1 \\
Mean dialysis duration pre-transplantation 382.6 \pm 233.2 days. \\
(preemptive: 4 cases) \\
Donor factors \\
Age at donation (years) \\
Gender (M/F) \\
Relation \\
Father \\
Brother \\
Sister \\
Husband
\end{tabular}

com $\mathrm{ABO}$ compatible, incom $\mathrm{ABO}$ incompatible, $\mathrm{mm}$ minor mismatch

Okinoerabu and Tokunoshima Islands, lying south of Kagoshima, are situated in the southern area of the Amami Islands (consisting of eight islands), about $100 \mathrm{~km}$ north of Okinawa and about $500 \mathrm{~km}$ south of Kyushu. Each of the two islands has only one general hospital, and the number of full-time physicians working for the general hospitals is only four in Tokunoshima Island and three in Okinoerabu Island. Their specialties are limited to general practice, surgery, gynecology and obstetrics, and pediatrics. Some other specialty outpatient departments are dependent on non-full-time physicians going to the islands from the mainland. In the case of specialty outpatient clinics for which no physicians go to the islands, such as transplant clinics as discussed in this article, it is necessary to travel to the mainland for each medical treatment. In the case of tertiary emergency, there are cases in which patients are transported to the mainland by air ambulance helicopter.

There are no facilities that specialize in kidney transplants, so the patients need to leave the island to receive the kidney transplantation, and up to a few years ago, there were less than five transplant patients on the island.

The transplant patients living in these islands had to go to the hospitals in Japan's mainland regularly by means of transportation such as airplane. For such patients, we cooperated with the physicians at the general hospital in these islands to newly establish a transplant outpatient department in the hospital. We visit this island from Osaka twice a month and examine the transplant patients at the outpatient department. As a result, the transplant patients living in these islands no longer have to travel a long distance to go to hospital.

We were able to reduce the burden on the patients by opening the transplant outpatient department in this island. This made it possible for the patients to feel kidney transplantation more familiar. We aim to have the transplant patients think that transplantation is one of the selectable renal replacement therapies by doing this.

The specific ways to do this are as follows.

Urinalysis and blood sampling were performed once a month. Allograft biopsy was performed in the diagnostic workup of graft dysfunction and proteinuria, or as protocol biopsy. We perform the biopsy there and evaluate and diagnose the biopsy specimens.

As shown in Fig. 1, renal transplant surgery and postoperative acute phase were performed at a transplantspecialized facility outside of the island. After a patient recovered in stable condition, the patient returned to his/ her own island and was followed up one or two times a month by a transplant physician. The transplant physician performed regular blood sampling before the consult; whenever an abnormal episode was observed, it was reported immediately to our hospital. As for treatment requiring specialized medical knowledge, the treatment was consulted and decided by local doctors and test results were sent to our hospital: in event of emergency, all such communications were conducted by phone or e-mail (Fig. 1). Based on that information, we diagnose the condition and guide the local doctors for treatment.

\section{Results}

In 2009, we physicians specializing in kidney transplants formed an alliance with local facilities to create specialized outpatient facilities, and the number of transplant patients has gradually increased (Fig. 2). Recently, the likelihood of kidney transplantation has been much higher among these islands (Table 2). 


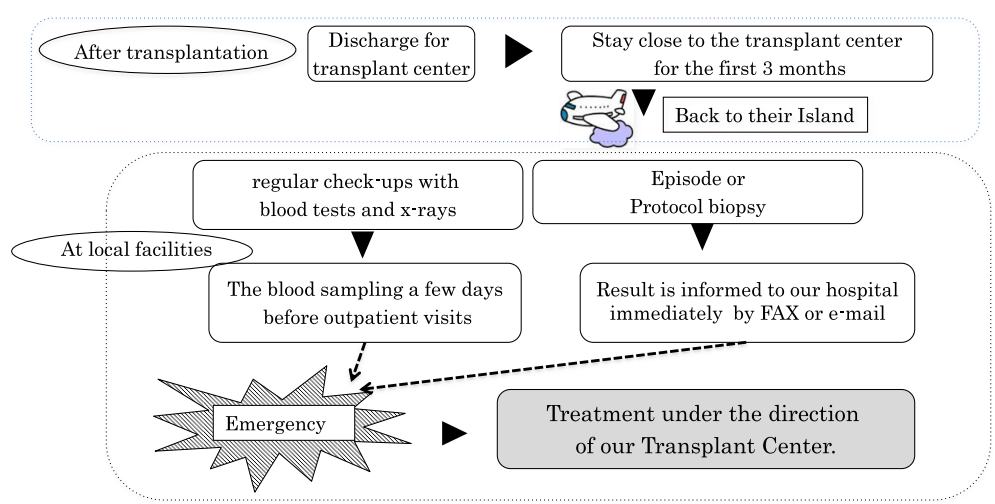

Fig. 1 Renal transplant surgery and post-operative acute phase are taken care of at the transplant specialized facility outside of the island. After the patient returns to the island and followed by a follow-up of one or two times a month by a transplant physician. The transplant physician takes a regular blood sampling and kidney biopsy beforehand, whenever an abnormal episode observed, it will be informed immediately to our hospital. The treatment is consulted and decided by local doctors and test results are sent to our hospital, all are conducted by phone or e-mail at an emergency

Delayed graft function was observed in only one patient, biopsy-proven acute rejection in four patients, and chronic allograft nephropathy in two patients. Post-transplant infections occurred in seven patients (Table 3). In these cases, the local doctor performed the treatment in the local facilities under our direction. Most of the treatments were performed safely and successfully.

Post-transplant lymphoproliferative disease (PTLD) occurred in one patient, and complete remission was achieved through dose reduction of immunosuppressive agents and administration of rituximab. The treatments were generally successful, with a mean follow-up period of $1208 \pm 1809$ days, and no patient had graft loss, with mean SCr of $1.35 \pm 0.85 \mathrm{mg} / \mathrm{dl}$.

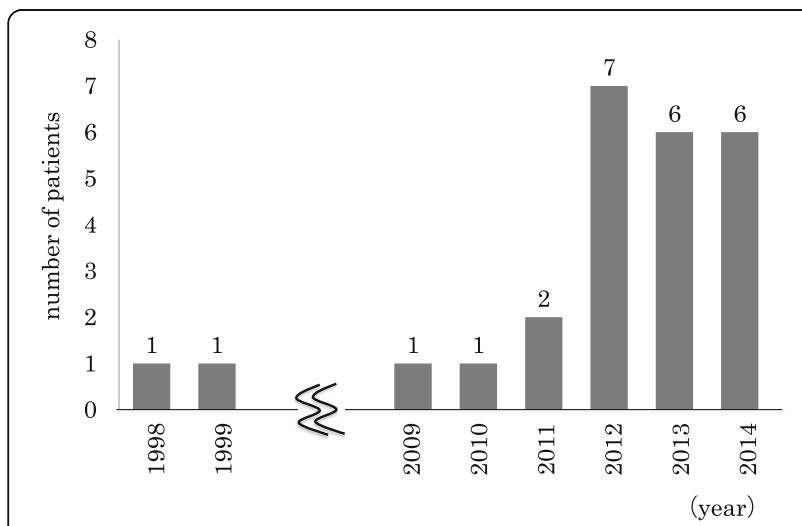

Fig. 2 In 2009, we physicians specializing in kidney transplants formed an alliance with local facilities to create specialized outpatient facilities, and the number of transplant patients has gradually increased

\section{Discussion}

Distance is a marker for survival after commencing dialysis in rural regions [1]. Transplantation is the best management option for most patients with end-stage renal disease. The likelihood of kidney transplantation was lower among remote- or rural-dwelling patients treated for kidney failure in the USA [2]. A previous study found that the likelihood of kidney transplantation among Canadian patients receiving dialysis was not influenced by distance from the closest transplant center [3]. A study from the USA found that post transplantation clinical outcomes were similar for those living closer to versus farther from a transplant center [4].

On Okinoerabu and Tokunoshima Islands, there were no facilities that specialized in kidney transplants. Access to health-care providers was a significant problem for these patients. Despite a geographic barrier to kidney transplantation, data on how remote residence location influences access to this essential medical service are sparse.

In this paper, we focused on an inconvenience regarding provision of transplant medical care on remote islands. However, for example, a similar situation would be considered for people living in rural areas of the Hokkaido prefecture. In Hokkaido, the clinical outcomes of

Table 2 Comparison of the number of transplant patients. Likelihood of renal transplantation has been much higher among these islands

\begin{tabular}{llll}
\hline & Tokunoshima & Okinoerabu & Japan \\
\hline Population & 27,000 & 13,700 & $127,799,000$ \\
HD patients & 43 & 39 & 304,592 \\
Renal transplant patients & 16 & 9 & 1276 \\
\hline
\end{tabular}

An Overview of Regular Dialysis Treatment in Japan (As of 31 December 2011) Factbook2011: The Japan Society for Transplantation 
Table 3 Complications after kidney transplant

\begin{tabular}{l}
\hline Complications after kidney transplant \\
\hline Rejection; 6 \\
Acute cellular rejection; 4 (Borderline change 2, \\
Acute T cell-mediated rejection 2) \\
Acute antibody-mediated rejection; 1 \\
Chronic active antibody-mediated rejection; 1 \\
Infection; 7 \\
Adeno virus 1 \\
Cytomegalovirus viremia 2 \\
BK virus 2 (viremia 1, nephropathy 1) \\
Urinary tract infections 2 \\
Others \\
Post-transplantation Lymphoproliferative Disorder 1
\end{tabular}

patients who received TKX and are living in a rural area are not worse than of those living in a city. The point of largest difference is whether the distance is land-based or sea-separated.

As a means of transportation to the main island of Okinawa, only one ferry is available per day and it takes 8 to $10 \mathrm{~h}$ to reach there from Okinoerabu or Tokunoshima Island. An additional 18 to $20 \mathrm{~h}$ are required to reach mainland Kyushu by ferry. For transportation by air, commercial flight service connecting Okinoerabu or Tokunoshima with Okinawa was abolished a few years earlier. The only flight route connecting to mainland Kagoshima provided just 2 to 4 flights per day. Furthermore, expensive airfares imposed a considerable financial burden on residents on the islands. Compared to those in remote areas on the mainland that are accessible by land, residents on the islands had far fewer choices for transportation and were at a great disadvantage in terms of travel expenses. In addition, there was no transportation available during the nighttime. Taking transplant patients as an example, there were cases in which some went to specialty hospitals on the mainland only once every 3 months and could not obtain medical treatment when a slight change occurred in their physical condition, resulting in their symptoms being left untreated.

These patients needed to leave the island to receive the procedure, which is a potential barrier to transplantation. We had been aware of such barriers, so we physicians specializing in kidney transplants formed an alliance with local facilities a few years back to create specialized outpatient facilities, and the number of transplant patients has gradually increased.

\section{Conclusions}

In conclusion, to coordinate medical care with primary care physicians, we physicians specializing in kidney transplants no longer need to travel long distances to receive follow-up outpatients. Our approach could be an effective way to promote a medical system for renal transplant surgery on other remote rural island regions in the near future.

\section{Abbreviations}

ADPKD: Autosomal dominant polycystic kidney disease; CYA: Cyclosporine; ESRD: End-stage renal disease; HLA: Human Leukocyte Antigen; KTX: Kidney transplantation; MMF: Mycophenolate mofetil; MP: Methylprednisolone; MZ: Mizoribine; SD: Standard deviation; TAC: Tacrolimus

\section{Acknowledgements \\ None. \\ Financial support \\ None.}

Availability of data and materials

Data will not be shared.

Authors' contributions

$\mathrm{HH}$ planned the study, searched the literature, and prepared the article. RM, $\mathrm{TM}, \mathrm{HU}, \mathrm{HN}$, and $\mathrm{HA}$ assisted in the article preparation. TT, NT, KS, TU, NI, and $\mathrm{Tl}$ participated in the design and coordination. $\mathrm{HH}$ and $\mathrm{YT}$ had participated to the Ethics Committee held in Okinoerabu Tokushukai hospital. All authors read and approved the final manuscript.

Ethics approval and consent to participate

This prospective observational study was approved by the Ethics Review Board of Okinoerabu Tokusyukai Hospital, and the study protocol conformed to the provisions outlined in the Declaration of Helsinki.

\section{Consent for publication}

Written informed consent was obtained from the patient for publication of this case report and any accompanying images.

\section{Competing interests}

The authors declare that they have no competing interests.

\section{Publisher's Note}

Springer Nature remains neutral with regard to jurisdictional claims in published maps and institutional affiliations.

\section{Author details}

'Department of Urology, Osaka Medical College, 2-7 Daigaku-machi, Takatsuki-shi, Osaka 569-8686, Japan. ²Okinoerabu Tokusyukai Hospital, Kagoshima, Japan.

Received: 24 October 2016 Accepted: 15 August 2017

Published online: 22 September 2017

References

1. Gray NA, Dent H, McDonald SP. Renal replacement therapy in rural and urban Australia. Nephrol Dial Transplant. 2012;27:2069-1969.

2. Tonelli M, Klarenbach S, Rose C, et al. Access to kidney transplantation among remote- and rural-dwelling patients with kidney failure in the United States. JAMA. 301(16):1681-90.

3. Tonelli M, Klarenbach S, Manns B, et al. Alberta kidney disease network. Residence location and like- lihood of kidney transplantation. CMAJ. 2006; 175(5):478-82

4. Kasiske BL, Snyder JJ, Skeans MA, Tuomari AV, Maclean JR, Israni AK. The geography of kidney trans- plantation in the United States. Am J Transplant 2008:8(3):647-57. 